\title{
12-Month Retrospective Comparison of Kahook Dual Blade Excisional Goniotomy with Istent Trabecular Bypass Device Implantation in Glaucomatous Eyes at the Time of Cataract Surgery
}

\author{
Mohammed K. ElMallah • Leonard K. Seibold • Malik Y. Kahook • \\ Blake K. Williamson · Inder P. Singh · Syril K. Dorairaj on behalf of the KDB Goniotomy Study Group
}

Received: June 5, 2019 / Published online: July 17, 2019

(C) The Author(s) 2019

\section{ABSTRACT}

Purpose: To compare the efficacy and safety of combined cataract extraction and either excisional goniotomy performed with the Kahook Dual Blade (KDB; phaco-KDB group) or single iStent trabecular bypass implantation (phacoiStent group) in eyes with mild to moderate glaucoma and visually significant cataract.

Methods: This was a retrospective analysis of 315 eyes from 230 adults with mild or moderate glaucoma treated with one or more intraocular pressure (IOP)-lowering medications (190 eyes of 134 subjects in the phaco-KDB group and 125 eyes of 96 subjects in the phaco-iStent group)

Enhanced Digital Features To view enhanced digital features for this article go to https://doi.org/10.6084/ m9.figshare.8343965.

M. K. ElMallah

Ocala Eye, Ocala, FL, USA

L. K. Seibold · M. Y. Kahook

School of Medicine, University of Colorado, Denver, CO, USA

B. K. Williamson

Williamson Eye Center, Baton Rouge, LA, USA

I. P. Singh

The Eye Center of Racine and Kenosha, Kenosha, WI, USA

S. K. Dorairaj ( $\square)$

Department of Ophthalmology, Mayo Clinic, Jacksonville, FL, USA

e-mail: syrildorairaj@gmail.com that required no subsequent surgical intervention for IOP control through 12 months of follow-up. Data included best-corrected visual acuity (BCVA), IOP, and IOP-lowering medications, collected preoperatively and at 1 week and $1,3,6$, and 12 months postoperatively as well as intraoperative and postoperative adverse events. The primary efficacy outcomes were the proportion of subjects in each group achieving $\geq 20 \%$ IOP reduction and $\geq 1$ medication reduction at month 12 . Subgroup analysis by baseline IOP ( $\leq 18 \mathrm{mmHg}$ vs. $>18 \mathrm{mmHg}$ ) was also performed.

Results: Mean (standard error) baseline IOP was $18.2(0.3) \mathrm{mmHg}$ in the phaco-KDB group and $16.7(0.3) \mathrm{mmHg}$ in the phaco-iStent group $(p=0.001)$. Statistically significant mean IOP and mean IOP medication reductions from baseline were achieved at all time points in both groups. Mean IOP reductions were significantly greater in the phaco-KDB group than in the phaco-iStent group at all time points including month 12 [- $5.0(0.3) \mathrm{mmHg}$ vs. $-2.3(0.4)$ $\mathrm{mmHg}, \quad p<0.001]$, while mean medication reductions were similar between groups at all time points except week 1 , when greater mean medication reduction was seen in the phacoiStent group ( -1.23 vs. -0.60 medications, $p<0.001$ ). At month 12 , IOP reductions $\geq 20 \%$ were achieved by $64.2 \%$ and $41.6 \%(p<0.001)$ in the phaco-KDB and phaco-iStent groups, respectively, and IOP medication reductions of $\geq 1$ medication were achieved by $80.4 \%$ and 
$77.4 \% \quad(p=0.522), \quad$ respectively. Intraocular pressure subgroup analysis revealed significant reductions in IOP-lowering medications without compromise of IOP control in lower IOP subgroups and significant reductions in both IOP and IOP-lowering medications in the higher IOP subgroups. The most common adverse events were transient IOP elevations and transient anterior chamber inflammation, which occurred with similar frequency in both groups and resolved spontaneously.

Conclusion: Goniotomy with the KDB lowered IOP significantly more than iStent implantation, with few adverse events in both groups. In eyes with mild to moderate glaucoma undergoing combined cataract extraction and glaucoma surgery, goniotomy with the KDB can safely deliver statistically significant and clinically meaningful reductions in both IOP and IOP medication burden through 12 months of follow-up.

Funding: New World Medical, Inc., provided funding for the study, medical writing assistance, Rapid Service Fees, and the open access fee.

Keywords: Angle surgery; Cataract extraction; Glaucoma; Goniotomy; Intraocular pressure; iStent; Kahook Dual Blade; MIGS; Trabecular meshwork

\section{INTRODUCTION}

The traditional stepped approach to glaucoma therapy-beginning with medications, then laser, then trabeculectomy, then tube-shunt surgery-has been challenged in recent years by both innovative clinical trials [1-4] and the development of minimally invasive glaucoma surgeries (MIGS) that offer significant reductions in intraocular pressure (IOP) with fewer adverse events than do more traditional surgical procedures [5-8]. Collectively, MIGS procedures are characterized as being effective, safe, and performed via minimally invasive techniques using an $a b$ interno approach with minimal disruption of normal ocular anatomy and physiology and rapid visual recovery [9]. These attributes favor pairing these procedures with cataract surgery to simultaneously improve visual acuity and lower IOP and/or the IOP medication burden in eyes with coexisting cataract and glaucoma. Many MIGS procedures have been approved in the US and other global markets for IOP reduction, some of which are specifically restricted to use in combination with cataract surgery.

MIGS procedures generally fall into one of three classes, depending on whether aqueous humor is shunted to Schlemm's canal, the supraciliary space, or the subconjunctival space. Excisional goniotomy using the Kahook Dual Blade (KDB; New World Medical, Inc, Rancho Cucamonga, CA) is a Schlemm's canal procedure. The KDB is designed to manually excise a strip of trabecular meshwork (TM) via an $a b$ interno approach through a previously created clear corneal incision. The instrument has a pointed distal tip that pierces the TM and enters Schlemm's canal and, as it is advanced along the trajectory of the canal, lifts and stretches the TM up the instrument's ramp and onto two parallel blades that excise a narrow strip of TM. Unlike a standard goniotomy knife that simply incises the $\mathrm{TM}$, leaving contiguous anterior and posterior flaps, the KDB excises a strip of TM, leaving a direct opening for aqueous to pass from the anterior chamber into Schlemm's canal. Thus, the KDB procedure removes the tissue at the site of aqueous outflow obstruction in open-angle glaucoma, restoring the natural aqueous outflow pathway without the formation of a filtering bleb and without the permanent implantation of a foreign object. In clinical studies, excisional goniotomy with the KDB lowered IOP 24-36\% and medication burden by $37-70 \%$ through 6-12 months of follow-up when performed as a standalone procedure or in combination with cataract surgery [10-13].

Excisional goniotomy with the KDB can be performed as a standalone procedure or in combination with cataract surgery. Other MIGS procedures approved for use in the US in combination with cataract surgery include the iStent and iStent Inject trabecular bypass devices (Glaukos, Inc, San Clemente, CA), the Hydrus microstent (Ivantis, Inc, Irvine, CA), and the CyPass supraciliary micro-stent (Alcon Laboratories, Inc., Fort Worth, TX), the latter of 
which was voluntarily withdrawn from the global marketplace over safety concerns related to long-term corneal endothelial cell loss [14]. The current comparative study utilized the iStent as the active control intervention. The iStent is a snorkel-shaped heparin-coated titanium device designed to be inserted through the TM, also via an ab interno approach through a clear corneal incision, so that its long arm rests within Schlemm's canal and its short arm traverses the TM and allows aqueous humor to enter from the anterior chamber, through the device, and into Schlemm's canal. The iStent is approved by the US FDA for use only in combination with cataract surgery. In clinical trials, the iStent lowered IOP and reduced reliance on IOP-lowering medications more than cataract surgery alone [15].

In this study, the 12-month IOP reduction and IOP-medication reduction achieved with KDB excisional goniotomy are compared with those seen with iStent implantation in eyes with coexisting glaucoma undergoing elective cataract extraction.

\section{METHODS}

This was a retrospective analysis of clinical data drawn from the health records of patients undergoing combined cataract and glaucoma surgery. No protected health information as defined by the health information portability and accounting act was collected [16], and the study was conducted under a waiver of consent following review by a central ethics board (Sterling IRB, Atlanta, GA). All procedures performed in studies involving human participants were in accordance with the ethical standards of the institutional and/or national research committee and with the 1964 Declaration of Helsinki and its later amendments or comparable ethical standards. The study was conducted under a waiver of consent following review of a central ethics board (Sterling IRB, Atlanta, GA).

Data were collected from adult subjects (at least 18 years of age) diagnosed with both visually significant cataract and mild to moderate glaucoma, defined as having visual field loss in only one hemifield and not within $5^{\circ}$ of fixation with corresponding optic nerve damage [17], treated with one or more topical IOP-lowering medications, who underwent elective phacoemulsification cataract extraction followed by either goniotomy with the Kahook Dual Blade (phaco-KDB group) or implantation of a single iStent trabecular bypass device (phaco-iStent group). Only eyes with uncomplicated phacoemulsification surgery [no vitreous loss, vitrectomy, or intraocular lens (IOL) implantation outside the lens capsule] and followed for 12 months with no subsequent IOP-lowering surgical procedures were included. Eyes were excluded for ocular comorbidities limiting potential postoperative visual gains (such as neovascular age-related macular degeneration, optic atrophy, etc.) or any prior history of incisional glaucoma surgery. Eyes that underwent additional IOP-lowering surgery during the follow-up period were also excluded from analysis.

As this was a retrospective analysis, no formal protocol governed procedure selection or surgical technique. Procedure selection was at each surgeon's discretion, and each procedure was performed as recommended by the respective manufacturers following completion of the cataract extraction and IOL implantation procedure. Postoperative use of anti-inflammatory, anti-infective, and miotic therapies was at each investigator's discretion. Baseline data were collected from a preoperative visit up to 6 weeks before surgery; intraoperative data and postoperative data at 1 week and $1,3,6$, and 12 months postoperatively were also collected. Data included demographics, preoperative glaucoma status, IOP, IOP medications, visual acuity, and adverse events at each time point. Intraocular pressure was measured using Goldmann applanation tonometry, and distance visual acuity was measured using the Snellen acuity chart at the 20 -foot equivalent distance under mesopic lighting.

The primary efficacy end points were reductions from baseline of IOP by $\geq 20 \%$ and IOP medications by $\geq 1$ medication. Mixed-modeling techniques to account for fellow-eye correlation were used to compare outcomes between groups using Bonferroni's correction to address multiplicity. In these patients undergoing elective cataract surgery, the rationale for adding a 
glaucoma procedure may have been to achieve a lower IOP, to reduce reliance on medications, or a combination of both. Those for whom the goal was further IOP reduction likely had higher baseline IOP, while those for whom the goal was medication reduction likely had lower baseline IOP. Given the retrospective nature of this analysis, these data were not recorded. To estimate the success of meeting these distinct goals, the analysis described above was repeated after dividing the entire sample into two groups based on the overall sample median of $18 \mathrm{mmHg}$ : those with baseline IOP $\leq 18 \mathrm{mmHg}$ and those with baseline IOP of $>18 \mathrm{mmHg}$. Medication reduction in the lower baseline IOP group in this cohort likely estimates the medication reduction expected in eyes controlled with medications at the time of surgery, while IOP reduction in the higher baseline IOP group likely estimates the IOP reduction expected in eyes inadequately controlled on medications at the time of surgery. Safety end points included postoperative BCVA, the incidence of ocular adverse events, and the incidence of secondary surgical interventions. Descriptive safety data are presented. Means are reported with standard error in parentheses (SE). This was designed as a descriptive study rather than to test a specific hypothesis; therefore, no formal power calculations were conducted a priori.

\section{RESULTS}

Data for this analysis were drawn from 315 eyes of 230 subjects, 134 subjects/190 eyes in the phacoKDB group and 96 subjects/125 eyes in the phaco-iStent group, operated upon by 13 experienced anterior segment surgeons. Demographic and glaucoma diagnosis data are given in Table 1. There were significantly fewer Caucasians (38.8\% vs. $64.6 \%$, respectively) and significantly more eyes with secondary glaucoma ( $27.9 \%$ vs. $14.4 \%$, respectively) in the phaco-KDB group compared with the phaco-iStent group.

\section{IOP Outcomes}

The proportion of patients achieving IOP reduction $\geq 20 \% \quad$ (Table 2 and Fig. 1) was

Table 1 Demographic and glaucoma diagnosis data

\begin{tabular}{|c|c|c|c|c|}
\hline & & $\begin{array}{l}\text { Phaco-KDB ( } n=190 \\
\text { eyes of } 134 \text { subjects) }\end{array}$ & $\begin{array}{l}\text { Phaco-iStent }(n=125 \\
\text { eyes of } 96 \text { subjects) }\end{array}$ & $p$ value \\
\hline Age (years) [mean (SE)] & & $69.6(0.8)$ & $72.4(0.8)$ & 0.014 \\
\hline \multirow[t]{2}{*}{ Gender $[n(\%)]$} & Female & $80(59.7)$ & $54(56.3)$ & 0.601 \\
\hline & Male & $54(40.3)$ & $42(43.8)$ & \\
\hline \multirow[t]{5}{*}{ Ethnicity $[n(\%)]$} & Caucasian & $52(38.8)$ & $62(64.6)$ & 0.002 \\
\hline & Hispanic & $56(41.8)$ & $24(25.0)$ & \\
\hline & Black & $18(13.4)$ & $5(5.2)$ & \\
\hline & Asian & $6(4.5)$ & $5(5.2)$ & \\
\hline & Other & $2(1.5)$ & $0(0)$ & \\
\hline \multirow[t]{5}{*}{ Glaucoma type by eye $[n(\%)]$} & Primary open angle & $137(72.1)$ & $107(85.6)$ & $<0.001$ \\
\hline & Exfoliation & $15(7.9)$ & $2(1.6)$ & \\
\hline & Pigmentary & $15(7.9)$ & $2(1.6)$ & \\
\hline & Angle closure & $13(6.8)$ & $0(0)$ & \\
\hline & Other & $4(2.1)$ & $10(8.0)$ & \\
\hline
\end{tabular}

$K D B \quad$ Kahook Dual Blade 
Table 2 Proportion of eyes meeting threshold success criteria for intraocular pressure (IOP) and medication reduction at each study time point

\begin{tabular}{|c|c|c|c|c|c|c|c|c|}
\hline & \multicolumn{2}{|l|}{$N$} & \multicolumn{3}{|c|}{ IOP reduction $\geq 20 \%$ from baseline } & \multicolumn{3}{|c|}{ Medications reduced by $\geq 1$ from baseline } \\
\hline & KDB & iStent & KDB $[n(\%)]$ & iStent $[n(\%)]$ & $p$ value* & KDB [n (\%)] & iStent $[n(\%)]$ & $p$ value $^{*}$ \\
\hline Week 1 & 180 & 94 & $95(52.8)$ & $35(37.2)$ & 0.014 & $87(48.6)$ & $80(87.0)$ & $<0.001$ \\
\hline Month 1 & 180 & 92 & $111(61.7)$ & $33(35.9)$ & $<0.001$ & $157(87.2)$ & $82(88.2)$ & 0.822 \\
\hline Month 3 & 170 & 74 & $119(70.0)$ & $37(50.0)$ & 0.003 & $143(84.1)$ & $65(83.3)$ & 0.876 \\
\hline Month 6 & 190 & 116 & $116(61.1)$ & $55(47.4)$ & 0.020 & $150(79.0)$ & $88(75.9)$ & 0.529 \\
\hline Month 12 & 192 & 125 & $123(64.1)$ & $52(41.6)$ & $<0.001$ & $153(80.1)$ & $96(77.4)$ & 0.567 \\
\hline
\end{tabular}

${ }^{*}$ Pearson's $\chi^{2}$ test adjusted for Bonferroni corrections

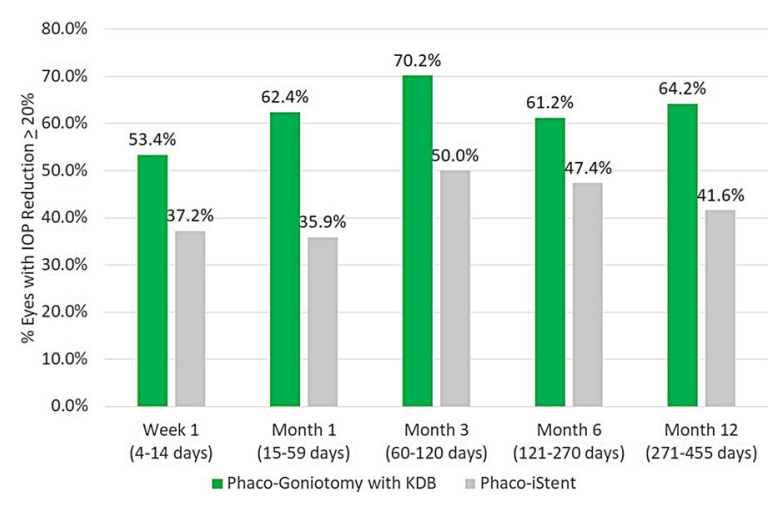

Fig. 1 Proportion of eyes with IOP reduction $\geq 20 \%$ in Phaco-Goniotomy with KDB and Phaco-iStent groups. Significant difference between groups at every time point $(p \leq 0.02)$

statistically greater in the phaco-KDB group at every time point $(p \leq 0.019)$. Across the five postoperative time points, IOP reductions of $\geq 20 \%$ from baseline were seen in $53.4-70.2 \%$ of eyes in the phaco-KDB group and in $37.2-50.0 \%$ of eyes in the Phaco-iStent group. At month $12,64.2 \%$ of phaco-KDB eyes and $41.6 \%$ of phaco-iStent eyes had IOP reductions $\geq 20 \%(p<0.001)$.

Baseline IOP was significantly higher in the phaco-KDB group compared with the phaco-iStent group [18.2 (0.3) vs. $16.7(0.3) \mathrm{mmHg}$, $p=0.001]$. Mean IOP reductions from baseline (Table 3 and Fig. 2) were statistically greater in the phaco-KDB group at all postoperative time points $(p \leq 0.007)$. In the phaco-KDB group, statistically significant reductions in IOP from baseline were seen at each of the five postoperative time points, ranging from -3.1 to
- $5.2 \mathrm{mmHg}$ (16.3-27.5\%; $p<0.001$ for each). In the phaco-iStent group, statistically significant IOP reductions from baseline were achieved at the month 1-6 time points ( $p$ $\leq 0.011$ ), but IOP reduction at week 1 was not significant $(-1.0 \mathrm{mmHg}, p=0.142)$. At month 12 , mean IOP reduction was greater in the phaco-KDB group than in the phaco-iStent group $[-5.0 \mathrm{mmHg}(-27.5 \%)$ vs. $-2.3 \mathrm{mmHg}$ $(-13.7 \%), p<0.001]$.

\section{Medication Outcomes}

The proportion of patients achieving IOP medication reduction $\geq 1$ medication (Table 2 ) was comparable in both groups at all time points except week 1, when more eyes in the phacoiStent than the phaco-KDB group were using $\geq 1$ fewer medication ( $87 \%$ vs. $48.6 \%$, respectively; $p<0.001)$. Across the five postoperative time points, IOP medication reductions from baseline of $\geq 1$ medication were seen in $48.6-87.6 \%$ of eyes in the phaco-KDB group and in $75.9-88.2 \%$ of eyes in the Phaco-iStent group. At month $12,80.4 \%$ of phaco-KDB eyes and $77.4 \%$ of phaco-iStent eyes had IOP medication reductions $\geq 1$ medication $(p=0.567)$.

Mean IOP medication reductions from baseline (Table 4 and Fig. 3) were comparable in both groups at all time points except week 1 , when the phaco-iStent group had greater medication reductions than the phaco-KDB group ( -1.23 vs. -0.60 medications; $p<0.001)$. In the phaco-KDB group, statistically significant reductions from baseline in IOP medications 


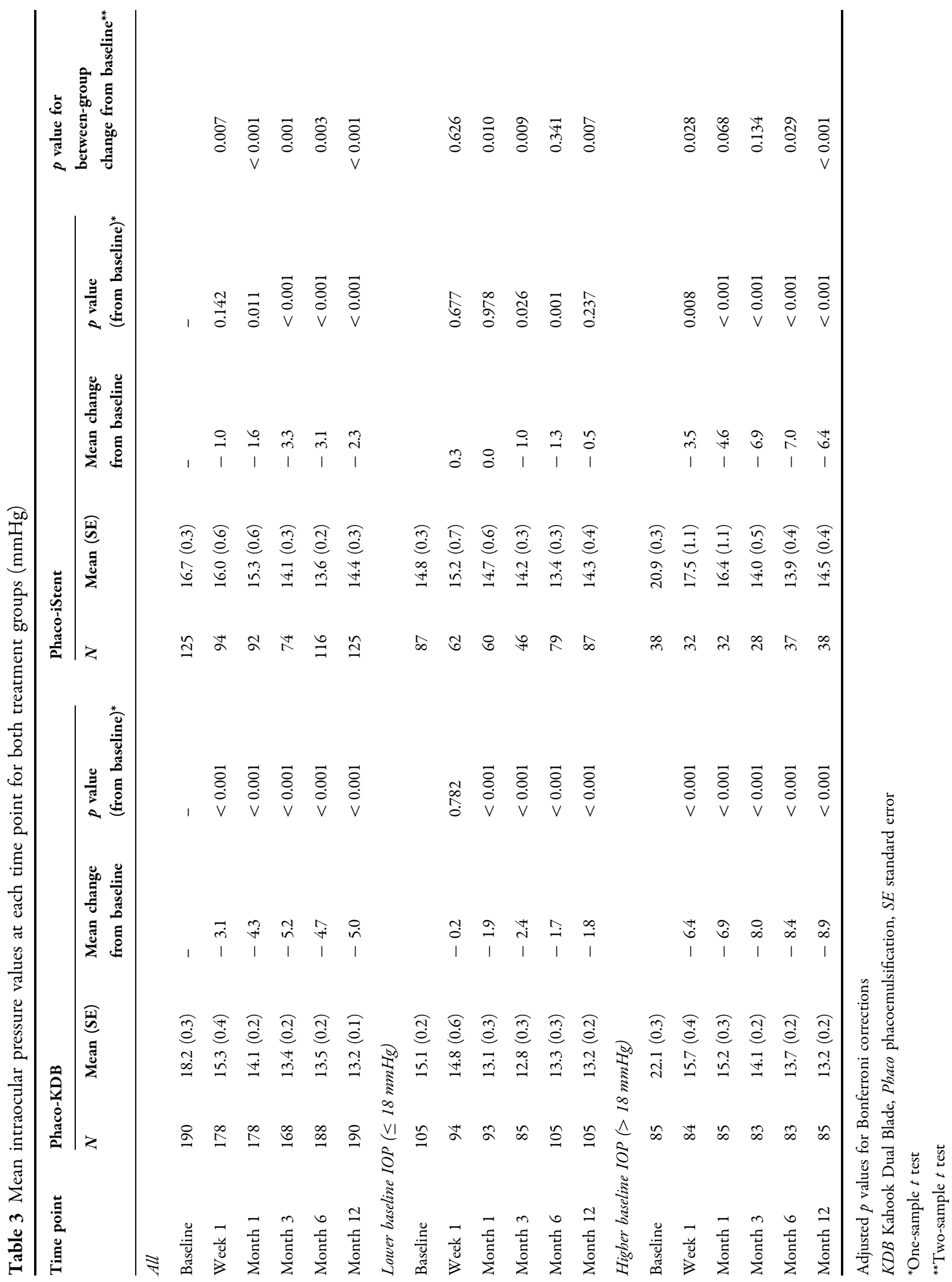




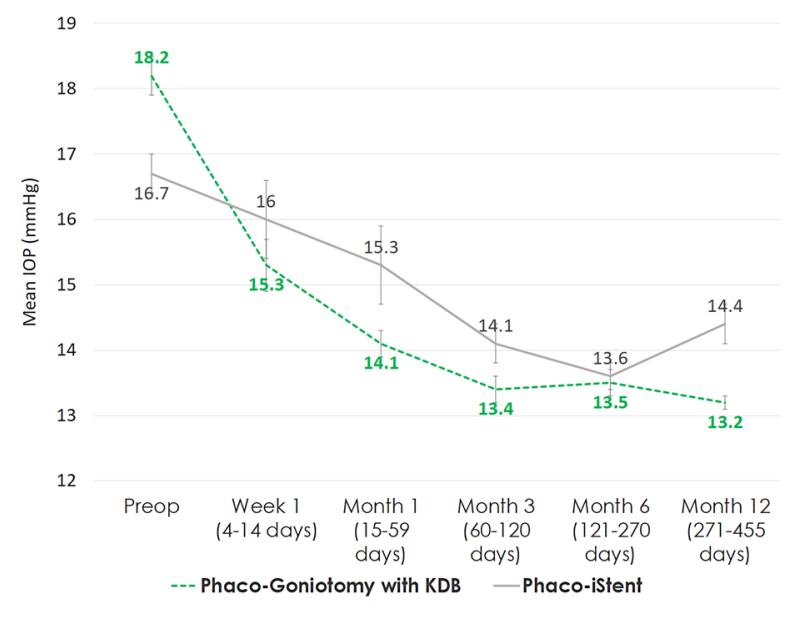

Fig. 2 Mean IOP at each time point for PhacoGoniotomy with KDB and Phaco-iStent groups (with standard error bars)

ranged from -0.60 to -1.10 medications $(p<0.001$ for all), while in the phaco-iStent group, reductions from baseline in IOP medications ranged from -0.93 to -1.23 medications and were also statistically significant at all time points $(p<0.001$ for all). At month 12 , mean IOP medication reductions were similar in both groups $[-1.03(71.7 \%)$ for phaco-KDB vs. $-0.97(63.2 \%), p=0.446]$.

\section{Outcomes in IOP Subgroups}

To better assess subject-specific goals, the efficacy analysis was repeated in two subgroups falling above and below the overall median of $18 \mathrm{mmHg}$ : those with baseline IOP $\leq 18 \mathrm{mmHg}$ and those with baseline IOP $>18 \mathrm{mmHg}$ (Tables 3 and 4). In the lower IOP groups, the primary goal of surgery was assumed to be medication reduction. In the lower IOP phacoKDB group, medications were reduced by a mean of -0.66 to -1.08 medications across time points $(p<0.001$ for all), with a mean medication reduction of $-0.94(0.08)$ medications at month 12 , at which time $74.3 \%$ of subjects had reduced their medication burden by $\geq 1$ medication. This was achieved with no compromise of IOP control, as mean IOP was reduced from baseline at every time point, including statistically significant IOP reductions of -1.7 to $-2.4 \mathrm{mmHg}$ at months $1-12$ $(p<0.001$ for all). In the low IOP phaco-iStent group, medications were reduced by a mean of -0.96 to -1.25 medications across time points $(p<0.001$ for all), with a mean medication reduction of $-1.00(0.08)$ medications at month 12 , at which time $80.2 \%$ of subjects had reduced their medication burden by $\geq 1$ medication. This was also achieved with no compromise of IOP control, as mean IOP was changed from baseline negligibly at most time points and reduced significantly only at months $3(-1.0 \mathrm{mmHg}, p=0.026)$ and $6(-1.3 \mathrm{mmHg}$, $p<0.001)$.

In the higher IOP groups, IOP reduction was likely the primary goal of surgery. In the highIOP phaco-KDB group, mean IOP reductions ranged from -6.4 to $-8.9 \mathrm{mmHg}(-28.9$ to - 40.4\%), with mean IOP at month 12 being reduced by $-8.9 \mathrm{mmHg}(-40.4 \%)$ and $97.7 \%$ of subjects achieving a minimum IOP reduction of $20 \%$. These IOP reductions were accompanied by significant reductions in mean medications ranging from -0.54 to -1.13 medications ( $p<0.001$ for all time points), with subjects using a mean of -1.13 fewer medications $(-82.4 \%)$ at month 12 . In the high-IOP phaco-iStent group, mean IOP reductions ranged from -3.5 to $-7.0 \mathrm{mmHg}(-16.2-33.6 \%)$, with mean IOP at month 12 being reduced by $-6.4 \mathrm{mmHg}(-30.6 \%)$ and $81.6 \%$ of subjects achieving a minimum IOP reduction of $20 \%$. These IOP reductions were accompanied by significant reductions in medications ranging from -0.86 to -1.19 medications $(p<0.001$ for all time points), with subjects using a mean of -0.92 fewer medications $(-59.3 \%)$ at month 12 .

\section{Visual Acuity Outcomes}

In the phaco-KDB group, BCVA improved from $0.34(0.02) \log$ MAR at baseline to $0.10(0.01)$ logMAR at month 12. In the phaco-iStent group, BCVA improved from 0.34 (0.03) logMAR at baseline to 0.10 (0.01) $\log$ MAR at month 12. No between-group difference in BCVA change from baseline was found at any time point $(p \geq 0.110)$. 


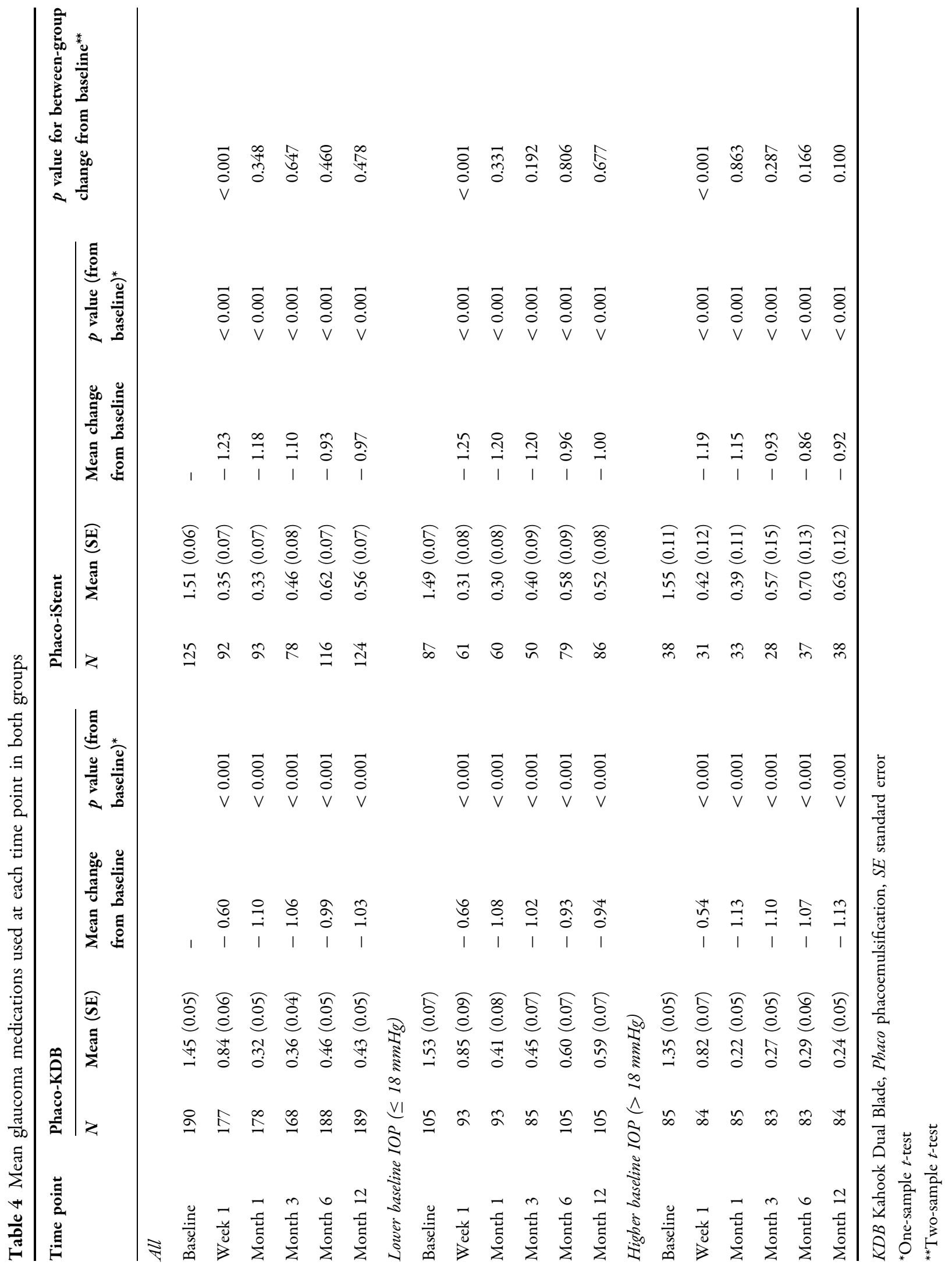




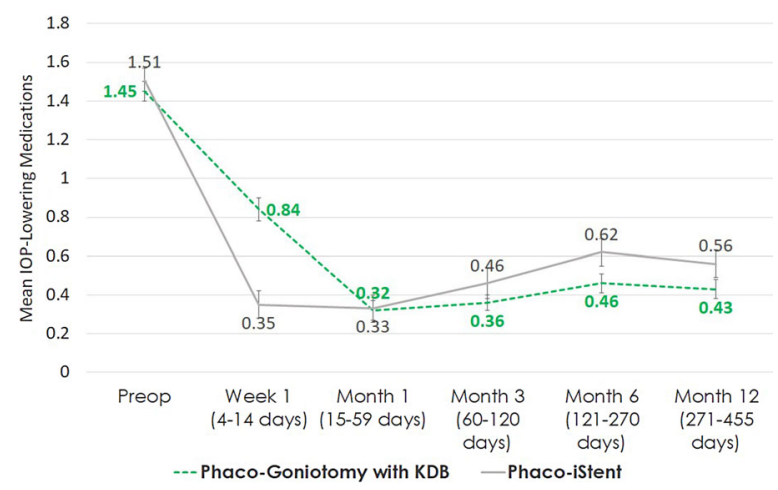

Fig. 3 Mean medication use at each time point for PhacoGoniotomy with KDB and Phaco-iStent groups (with standard error bars)

Table 5 Incidence of adverse events occurring in $\geq 1 \%$ of the total sample by treatment group, $n(\%)$

\begin{tabular}{lll}
\hline Adverse event & $\begin{array}{l}\text { Phaco-KDB } \\
(\boldsymbol{n}=\mathbf{1 9 0} \text { eyes })\end{array}$ & $\begin{array}{l}\text { Phaco-iStent } \\
(\boldsymbol{n}=\mathbf{1 2 5} \text { eyes })\end{array}$ \\
\hline $\begin{array}{c}\text { Transient anterior } \\
\text { chamber }\end{array}$ & $2(1.0)$ & $3(2.4)$ \\
$\quad$ inflammation & & $1(0.8)$ \\
$\begin{array}{c}\text { Transient IOP } \\
\text { elevation }\end{array}$ & $2(1.0)$ & $1(0.8)$ \\
$\begin{array}{l}\text { Corneal edema } \\
\text { Posterior capsule } \\
\text { opacification }\end{array}$ & $2(1.0)$ & $1(0.8)$ \\
\hline
\end{tabular}

KDB Kahook Dual Blade, Phaco phacoemulsification

\section{Safety Outcomes}

Adverse events occurring in $\geq 1 \%$ of the total sample are given in Table 5. Adverse events were generally mild to moderate in intensity and resolved spontaneously. The most common adverse events were transient anterior chamber inflammation during the first postoperative week, occurring in two (1.0\%) phaco-KDB eyes and three $(2.4 \%)$ phaco-iStent eyes, and transient IOP elevation during the 1st postoperative week, occurring in two (1.0\%) phaco-KDB eyes and one $(0.8 \%)$ phaco-iStent eye. Other adverse events occurring in $1 \%$ or more of eyes included corneal edema and posterior capsule opacification. Rates of these events were similar between groups. Transient blood reflux, which is an expected component of angle surgery and not an adverse event, was seen in 38 eyes in the phaco-KDB group (19.8\%) and in 5 eyes in the phaco-iStent group (4\%). Blood reflux cleared spontaneously in all cases.

\section{DISCUSSION}

In this retrospective comparative analysis, excisional goniotomy with the KDB provided greater IOP reduction than did iStent $(-5.0$ vs. $-2.3 \mathrm{mmHg}$, respectively; $p<0.001)$ at 12 months when both were performed in combination with cataract surgery. Furthermore, more eyes achieved IOP reductions of $\geq 20 \%$ at 12 months in the phaco-KDB group compared with the phaco-iStent group $(64.2 \%$ vs. $41.6 \%$, respectively; $p<0.001)$. When subject-specific goals were considered, 12-month mean IOP reductions in eyes with higher baseline IOP were $-8.9 \mathrm{mmHg}(-40.4 \%)$ and $-6.4 \mathrm{mmHg}$ $(-30.6 \%)$ in the phaco-KDB and phaco-iStent groups, respectively $(p<0.001)$, and mean medication reductions were $-0.94(-61.5 \%)$ and $-1.00(-65.0 \%)$ in the two lower baseline IOP subgroups, respectively $(p=0.677)$, with no adverse effects on IOP control.

The IOP reductions seen in this study are consistent with IOP outcomes reported in prior studies. In prospective and retrospective studies of excisional goniotomy with the KDB performed at the time of cataract surgery, mean IOP reductions of -24 to $-26.2 \%$ have been reported with follow-up periods of 6-12 months $[12,13]$. In these same studies, the IOP-lowering medication burden was reduced by -47.4 to $-50 \%[12,13]$. Outcomes in the current study, a reduction of $-27.3 \%$ in IOP and $-70.0 \%$ in IOP-lowering medications, are at least as favorable as in these prior reports. Likewise, studies of iStent implantation at the time of cataract surgery have reported IOP reductions in the range of -8.2 to $-20.2 \%$, with IOP medication reductions ranging from -22.1 to $-93.3 \%$, at 12-24 months [15, 18-20]. A meta-analysis of studies evaluating outcomes after combined cataract and iStent implantation concluded that the procedure delivered approximately $-9 \%$ 
IOP reduction after 1-5 years [21]. A retrospective single-site, single-surgeon comparison of KDB-Phaco vs. iStent-Phaco recently reported mean IOP reductions of $12.6 \%$ and $14.3 \%$, respectively, in a cohort of eyes with low ( $\sim 17.5 \mathrm{mmHg}$ baseline IOP) and mean medication reductions of $27 \%$ and $65 \%$, respectively, at 12 months [22]. These reported results are also consistent with the findings of the current study in which the phaco-iStent group had mean IOP reduction of $-13.7 \%$ and medication reduction of $-63.2 \%$. Therefore, the differences observed in this study favoring excisional goniotomy with the KDB over iStent are not attributable to overperformance of the KDB procedure or underperformance of the iStent procedure arising from case selection bias and likely represent a true therapeutic advantage of KDB excisional goniotomy over iStent implantation in terms of IOP reduction in eyes with mild or moderate glaucoma undergoing combined cataract and glaucoma surgery.

This superior IOP-lowering efficacy of the phaco-KDB procedure over the phaco-iStent procedure was achieved with no increase in adverse events compared with iStent implantation. The most common adverse events observed, anterior chamber inflammation and transient elevations of IOP, occurred with similar incidence in both groups, and other less common adverse events also occurred at equal rates in both groups. Blood reflux, common to most angle procedures [23-26], was more common in the phaco-KDB group likely because of the nature of the procedure, as trabecular meshwork is excised in this procedure vs. only opened in the iStent procedure. One safety advantage of KDB goniotomy over iStent is that no permanent device is implanted in the eye during KDB goniotomy. Unanticipated longterm safety issues have been identified with MIGS procedures that require permanent device implantation. In the 5-year COMPASS XT study, significantly greater changes in corneal endothelial cell density were observed at month 60 in eyes undergoing cataract plus CyPass implantation $(-20.4 \%)$ compared with eyes undergoing cataract surgery alone $(-10.1 \%$, $p=0.0032$ ) [27], leading the manufacturer to voluntarily withdraw the device from the global marketplace [14]. Also, the absence of a permanent indwelling device ensures no contraindication to magnetic resonance imaging (MRI) if indicated for any reason subsequent to surgery. The iStent device is rated MR Conditional (indicating that MRI can be safely performed under certain conditions) [28], but such safety has not been evaluated clinically and is specific to lower Telsa $(\leq 3 \mathrm{~T})$ MRI systems, while higher T systems (7 T [29] and above [30]) are being developed for clinical use and are known to have higher transient adverse event rates than lower T systems $[29,30]$.

This study is limited by its retrospective design, in which subjects were not randomly assigned to treatment groups and outcomes data were collected via routine clinical practice, which is typically less robust than in prospective clinical trials. Of note, baseline demographic characteristics were unbalanced between groups, with more ethnic minorities and more secondary glaucomas in the phacoKDB group. These are known risk factors for glaucoma surgery failure (although the effect of ethnicity on MIGS outcomes has not been evaluated and may differ from the effect known to affect traditional filtering surgeries [31]) and may have biased the study's findings in favor of the iStent group, thus underestimating the efficacy of KDB goniotomy. Conversely, as pigmentary, pseudoexfoliation, and angle-closure glaucomas directly affect the TM and may be more amenable to TM-based procedures, having more of these cases in the KDB group could bias results in its favor. In fact, a recent publication reported IOP reductions of $\sim 50 \%$ and medication reductions of $\sim 90 \% 6$ months after combined phacoemulsification, goniosynechialysis, and excisional goniotomy with the KDB in eyes with chronic angle closure glaucoma and peripheral anterior synechiae [32]. Also, mean baseline IOP was higher in the KDB group compared with the iStent group, which potentially permits a greater therapeutic effect. However, the $\sim 40-180 \%$ greater percent IOP reductions from baseline seen in the KDB group cannot be explained by the $\sim 8 \%$ difference in mean baseline IOP between the groups. Finally, only eyes that required no additional IOP-lowering surgery were included 
in this analysis. Thus, these results represent the outcomes to be expected in successful surgical cases and not in all cases undergoing these combined cataract-MIGS procedures. This provides a true estimate of efficacy in eyes with successful surgery, which can assist with procedure selection based on surgical goals of individual patients, but does not provide data on the likelihood of success (which have been reported elsewhere $[12,13,15,18-21])$.

In summary, goniotomy with the KDB lowered IOP significantly more than did iStent implantation, both procedures at the time of cataract surgery, with comparable safety profiles. In eyes with mild to moderate glaucoma undergoing combined cataract extraction and glaucoma surgery, goniotomy with the KDB can deliver statistically significant and clinically meaningful reductions in both IOP and IOP medication burden.

\section{ACKNOWLEDGEMENTS}

Funding. New World Medical, Inc., provided funding for the study, medical writing assistance, article processing charges, and the open access fee.

Editorial Assistance. We thank Anthony D. Realini, MD, MPH, for assisting in writing the manuscript. Dr. Realini, Hypotony Holdings, LLC, is an independent consultant to New World Medical. New World Medical provided funding for the medical writing assistance.

Authorship. All named authors meet the International Committee of Medical Journal Editors (ICMJE) criteria for authorship for this article, take responsibility for the integrity of the work as a whole, and have given their approval for this version to be published.

Disclosures. Mohammed K. ElMallah declares that he has received consulting fees and research support from New World Medical for the conduct of this study and research support from Glaukos, Ivantis, and Unity. Leonard Seibold declares that he has received consulting fees from New World Medical, was a consultant to New World Medical for the conduct of this study, and has received research support from New World Medical in the past. Blake K. Williamson declares that he has received consulting fees and research support from New World Medical for the conduct of this study and consulting fees from Alcon, Allergan, Bausch and Lomb, Biotissue, Diopsys, Glaukos, Johnson and Johnson Vision, Shire, Sight Sciences, and Zeiss. Inder Paul Singh declares that he has received consulting fees and research support from New World Medical for the conduct of this study. Malik Y. Kahook declares he has received consulting fees from Allergan, patent royalties and consulting fees from Alcon and New World Medical, and is the inventor of the KDB device. Syril K. Dorairaj declares that he has received consulting fees from New World Medical, was a consultant to New World Medical for the conduct of this study, and has received research support from New World Medical in the past.

Compliance with Ethical Guidelines. All procedures performed in studies involving human participants were in accordance with the ethical standards of the institutional and/or national research committee and with the 1964 Helsinki Declaration and its later amendments or comparable ethical standards. The study was conducted under a waiver of consent following review of a central ethics board (Sterling IRB, Atlanta, GA).

Data Availability. The datasets generated during and/or analyzed during the current study are available from the corresponding author on reasonable request. All authors had full access to all of the data in this study and take complete responsibility for the integrity of the data and accuracy of the data analysis. We thank the participants of the study.

Open Access. This article is distributed under the terms of the Creative Commons Attribution-NonCommercial 4.0 International License (http://creativecommons.org/licenses/ by-nc/4.0/), which permits any noncommercial use, distribution, and reproduction in any medium, provided you give appropriate credit 
to the original author(s) and the source, provide a link to the Creative Commons license, and indicate if changes were made.

\section{REFERENCES}

1. Katz LJ, Steinmann WC, Kabir A, Molineaux J, Wizov SS, Marcellino G. Selective laser trabeculoplasty vs medical therapy as initial treatment of glaucoma: a prospective, randomized trial. J Glaucoma. 2012;21:460-8.

2. Lichter PR, Musch DC, Gillespie BW, et al. Interim clinical outcomes in the Collaborative Initial Glaucoma Treatment Study comparing initial treatment randomized to medications or surgery. Ophthalmology. 2001;108:1943-53.

3. Gedde SJ, Schiffman JC, Feuer WJ, Herndon LW, Brandt JD, Budenz DL. Treatment outcomes in the Tube vs Trabeculectomy (TVT) study after 5 years of follow-up. Am J Ophthalmol. 2012;153:789-803.

4. Gedde SJ, Feuer WJ, Shi W, et al. Treatment outcomes in the Primary Tube vs Trabeculectomy Study after 1 year of follow-up. Ophthalmology. 2018;125:650-63.

5. Richter GM, Coleman AL. Minimally invasive glaucoma surgery: current status and future prospects. Clin Ophthalmol. 2016;10:189-206.

6. Agrawal P, Bradshaw SE. Systematic literature review of clinical and economic outcomes of microinvasive glaucoma surgery (MIGS) in primary openangle glaucoma. Ophthalmol Ther. 2018;7:49-73.

7. Chen DZ, Sng CCA. Safety and efficacy of microinvasive glaucoma surgery. J Ophthalmol. 2017;2017:3182935.

8. Bovee CE, Pasquale LR. Evolving surgical interventions in the treatment of glaucoma. Semin Ophthalmol. 2017;32:91-5.

9. Saheb H, Ahmed II. Micro-invasive glaucoma surgery: current perspectives and future directions. Curr Opin Ophthalmol. 2012;23:96-104.

10. Greenwood MD, Seibold LK, Radcliffe NM, et al. Goniotomy with a single-use dual blade: short-term results. J Cataract Refract Surg. 2017;43:1197-201.

11. Salinas L, Chaudhary A, Berdahl JP, et al. Goniotomy using the Kahook Dual Blade in severe and refractory glaucoma: 6 month outcomes. J Glaucoma. 2018;10:849-55.
12. Dorairaj SK, Kahook MY, Williamson BK, Seibold LK, ElMallah MK, Singh IP. A multicenter retrospective comparison of goniotomy vs trabecular bypass device implantation in glaucoma patients undergoing cataract extraction. Clin Ophthalmol (Auckl NZ). 2018;12:791-7.

13. Dorairaj SK, Seibold LK, Radcliffe NM, et al. 12-Month outcomes of goniotomy performed using the Kahook Dual Blade combined with cataract surgery in eyes with medically treated glaucoma. Adv Ther. 2018;35:1460-9.

14. Alcon announces voluntary global market withdrawal of CyPass Micro-Stent for surgical glaucoma. https://www.novartis.com/news/media-releases/ alcon-announces-voluntary-global-market-with drawal-cypass-micro-stent-surgical-glaucoma. Accessed 29 Aug 2018.

15. Samuelson TW, Katz LJ, Wells JM, Duh YJ, Giamporcaro JE, Group USiS. Randomized evaluation of the trabecular micro-bypass stent with phacoemulsification in patients with glaucoma and cataract. Ophthalmology. 2011;118:459-67.

16. National Institutes of Health. HIPAA privacy rule. https://privacyruleandresearch.nih.gov/pr_08.asp. Accessed 2 Oct 2018.

17. ICD-10-CM quick reference guide for glaucoma. San Franc: Am Acad Ophthalmol 2015.

18. Seibold LK, Gamett KM, Kennedy JB, et al. Outcomes after combined phacoemulsification and trabecular microbypass stent implantation in controlled open-angle glaucoma. J Cataract Refract Surg. 2016;42:1332-8.

19. Ferguson TJ, Berdahl JP, Schweitzer JA, Sudhagoni RG. Clinical evaluation of a trabecular microbypass stent with phacoemulsification in patients with open-angle glaucoma and cataract. Clin Ophthalmol. 2016;10:1767-73.

20. Tan SZ, Au L. Manchester iStent study: 3-year results and cost analysis. Eye (Lond). 2016;30:1365-70.

21. Malvankar-Mehta MS, Chen YN, Iordanous Y, Wang WW, Costella J, Hutnik CM. iStent as a solo procedure for glaucoma patients: a systematic review and meta-analysis. PLoS One. 2015;10:e0128146.

22. Le C, Kazaryan S, Hubbell M, Zurakowski D, Ayyala RS. Surgical outcomes of phacoemulsification followed by iStent implantation vs goniotomy with the Kahook Dual Blade in patients with mild primary open-angle glaucoma with a minimum of 12-month follow-up. J Glaucoma. 2019;28:411-4. 
23. Minckler DS, Baerveldt G, Alfaro MR, Francis BA. Clinical results with the trabectome for treatment of open-angle glaucoma. Ophthalmology. 2005;112:962-7.

24. Vold S, Ahmed II, Craven ER, et al. Two-year COMPASS trial results: supraciliary microstenting with phacoemulsification in patients with openangle glaucoma and cataracts. Ophthalmology. 2016;123:2103-12.

25. Popovic M, Campos-Moller X, Saheb H, Ahmed IIK. Efficacy and adverse event profile of the iStent and iStent inject trabecular micro-bypass for open-angle glaucoma: a meta-analysis. J Curr Glaucoma Pract. 2018;12:67-84.

26. Grover DS, Flynn WJ, Bashford KP, et al. Performance and safety of a new $\mathrm{Ab}$ interno gelatin stent in refractory glaucoma at 12 months. Am J Ophthalmol. 2017;183:25-36.

27. Lane S. Overview of the results from the 5 year follow up study of the CyPass microstent. European society for cataract and refractive surgery annual meeting. Vienna, Austria; 2018.
28. Glaukos Inc. iStent and iStent inject DFU and MRI information. https://www.glaukos.com/dfu-mriinformation/. Accessed 23 Apr 2019.

29. Karamat MI, Darvish-Molla S, Santos-Diaz A. Opportunities and challenges of 7 tesla magnetic resonance imaging: a review. Crit Rev Biomed Eng. 2016;44:73-89.

30. Budinger TF, Bird MD. MRI and MRS of the human brain at magnetic fields of $14 \mathrm{~T}$ to 20T: technical feasibility, safety, and neuroscience horizons. NeuroImage. 2018;168:509-31.

31. American Academy of Ophthalmology. Primary open-angle glaucoma: preferred practice pattern. San Francisco: American Academy of Ophthalmology; 2015.

32. Dorairaj S, Tam MD. Kahook Dual Blade excisional goniotomy and goniosynechialysis combined with phacoemulsification for angle closure glaucoma: 6-month results. J Glaucoma; 2019 (in press). 